Revista de Investigación Universitaria, 2016, Vol. 5 (1): 25-31

ISSN: 2312-4253 (Versión impresa) ISSN: 2078-4015 (Versión digital)

\title{
Relación entre la calidad de sueño con el grado de obesidad y circunferencia de cintura en comerciantes del Gran Mercado Mayorista de Lima, 2016

\author{
Relationship between quality of sleep with the degree of obesity and waist circumference \\ in merchants of the Great Wholesale Market of Lima, 2016
}

\author{
Plaza Vera, Karem Johanna; Ambulay Briceño, Johnny Percy \\ EP de Nutrición Humana, Facultad de Ciencias de la Salud, Universidad Peruana Unión \\ Recibido el 8 de setiembre del 2015 - Aceptado el 14 de noviembre del 2015
}

\begin{abstract}
Resumen
Objetivo: Determinar la relación entre la calidad de sueño con el grado de obesidad y circunferencia de cintura. Materiales y Métodos: Se realizó un análisis transversal en 198 comerciantes de 30 a 59 años: 116 mujeres y 86 varones. Se evaluó la calidad de sueño a través del índice de calidad de sueño Pittsburg; asimismo, se midió el peso, talla y circunferencia de la cintura en la ficha antropométrica para calcular el grado de obesidad y circunferencia de cintura y clasificarlo según la OMS. Resultados: En ambos sexos hay mayor prevalencia de obesidad tipo II y de riesgo aumentado de enfermedades cardiovasculares, así como una mala calidad de sueño. Finalmente, se observó una relación significativa entre la calidad de sueño con el grado de obesidad y circunferencia de cintura en las mujeres y no en los varones. Conclusión: Se sugiere que la mala calidad de sueño está relacionado con el aumento de riesgo a desarrollar obesidad y riesgo cardiometabólico.
\end{abstract}

Palabras clave: Calidad de sueño, obesidad, circunferencia de cintura.

\begin{abstract}
Objective: To determine the relationship between sleep quality and the degree of obesity and waist circumference. Materials and Methods: A cross-sectional analysis of 198 marketers aged 30 to 59 years was carried out, 116 women and 86 boys were evaluated for sleep quality through the Pittsburgh Sleep Quality Index, as well as weight, height and waist circumference In the anthropometric chart to calculate the degree of obesity and waist circumference and to classify it according to the WHO. Results: In both sexes there is a higher prevalence of type II obesity and an increased risk of cardiovascular diseases, as well as poor sleep quality. Finally, a significant relationship was observed between sleep quality and the degree of obesity and waist circumference in the Women and not in males. Conclusion: It is suggested that poor sleep quality is related to increased risk of developing obesity and cardio metabolic risk.
\end{abstract}

Keywords: Sleep quality. Obesity. Waist circumference.

\section{Introducción}

Diversos estudios han demostrado que la calidad de sueño está relacionada con la obesidad. El sueño es una necesidad humana básica. El número de horas necesarias que un individuo promedio debe dormir diariamente para mantener un funcionamiento adecuado durante la vigilia se ha definido en 7 horas. La calidad de sueño no solo está definido como el dormir bien durante la noche sino también tener un desempeño adecuado durante la vigilia (Granados et al. 2013).

La mala calidad de sueño y los trastornos asociados constituyen un problema de salud. Las personas subestiman la importancia del sueño en su vida diaria durmiendo menos horas de las recomendadas, perjudicando su salud

Correspondencia al autor:

email: karemplaza@upeu.edu.pe; johnnyambulay@upeu.edu.pe 
física y mental. Los riesgos de salud asociados a la falta de sueño son principalmente las deficiencias del sistema inmunológico, cardiovascular, y aumento de peso (Guevara 2013). Dormir adecuadamente ayuda al restablecimiento de la energía, eliminación de radicales libres acumulados durante el día, regulación y restauración de la actividad eléctrica cortical, regulación térmica, regulación metabólica y endocrina, homeostasis sináptica, activación inmunológica, consolidación de la memoria, entre otros (Carrillo P. \& Ramírez J. 2013).

La obesidad es un problema de salud pública por su prevalencia a nivel mundial, tanto en los países desarrollados como en países en vías de desarrollo (Álvarez D., Sánchez J. \& Gómez G. 2012). La Organización Mundial de la Salud define la obesidad como una enfermedad caracterizada por el aumento de la grasa corporal, asociada a un mayor riesgo para la salud (Moreno 2012).

Desde 1980 se ha duplicado en todo el mundo la tasa de obesidad, tal es así que en el 2014, 600 millones eran obesos y, se estima que para el 2020 más de dos terceras partes de la población mundial tendrá sobrepeso u obesidad (OMS 2015). Según el Instituto Nacional de Estadística e Informática (INEI), basándose en la Encuesta Demográfica y de Salud Familiar 2015 del Perú, el 17,5\% de las personas padecen obesidad, siendo el $22,4 \%$ mujeres y el $13.3 \%$ hombres. Los departamentos con mayor obesidad son Tacna con el $30,4 \%$, le siguen Tumbes e Ica con 26,2\%, Moquegua $25,7 \%$ y la Provincia Constitucional del Callao con 25,4\% (Instituto Nacional de Estadística e Informática 2016). Actualmente, en el Perú, más del $63 \%$ de la población entre 30 y 59 años sufren de sobrepeso y obesidad (Nutrición 2016).

La epidemia de la obesidad es considerada como el quinto factor de riesgo de muerte en el mundo, porque cada año mueren unos 2, 8 millones de personas adultas como consecuencia de sobrepeso u obesidad. Las causas asociadas a la obesidad son los malos hábitos alimentarios, conductuales, y las horas de sueño. Esta epidemia es responsable del $44 \%$ como desencadenante de diabetes, $23 \%$ de cardiopatías isquémicas y entre 7 y $41 \%$ con la aparición de algunos cánceres (Cascales 2015; Moreno 2012). Por otro lado, la circunferencia de la cintura es un indicador de la grasa visceral y problemas cardiovasculares (Carmenate et al. 2007).

Un estudio epidemiológico reporta que la falta de sueño aumenta la prevalencia de obesidad, ya que la relación entre la restricción del sueño, aumento de apetito y ganancia de peso podrían derivarse de alteraciones en el metabolismo de hormonas que regulan el apetito (grelina) y hambre (leptina), provocando una disminución del gasto energético (Cárdenas, Roandy, y Hernández 2012). En estudios realizados se encontró cómo la mala calidad de sueño puede predecir la obesidad (Rahe et al. 2015). En otro estudio realizado en personas de 30 a 65 años se encontró que en las mujeres la calidad de sueño se asoció con la obesidad, mientras que en los varones no (Bidulescu et al. 2010). Debido a la falta de estudios locales, el objetivo fue ver si existe relación entre la calidad de sueño con el grado de obesidad y circunferencia de cintura en comerciantes que duermen menos cantidades de horas por las características de actividades que realizan.

\section{Método}

Esta investigación es de enfoque cuantitativo, de corte transversal, de diseño no experimental y de tipo descriptivo correlacional. Los participantes fueron 116 mujeres y 86 varones obesos de 30-59 años, quienes fueron tomados del Gran Mercado Mayorista de Lima. Los participantes elegidos para participar fueron comerciantes con un IMC $>30$, excluyéndose a los que tomaban medicamentos para el insomnio y a quienes presentaban hipotiroidismo. Todos los participantes proveyeron el consentimiento informado por escrito como un requisito de la Universidad Peruana Unión.

A los participantes se les midió el peso, la talla y circunferencia abdominal (CA). El IMC se calculó dividiendo el peso sobre la talla al cuadrado $(\mathrm{Kg} / \mathrm{m} 2)$ para clasificarlos por grados de obesidad: grado 1, grado 2 y grado 3 . La CA entre $80-87 \mathrm{~cm}$ y $94-101$ $\mathrm{cm}$ fueron clasificados como riesgo aumentado para mujeres y varones respectivamente, y en el caso de $>88 \mathrm{~cm} \mathrm{y}>102 \mathrm{~cm}$ fue clasificado como riesgo alto para adquirir enfermedades cardiovasculares en el orden mencionado.

La calidad de sueño se evaluó utilizando el índice de calidad de sueño de Pittsburgh (ICSP) en el mes pasado. El ICSP tiene 19 preguntas que son agrupadas en siete componentes: calidad subjetiva del sueño, latencia subjetiva del sueño, duración del sueño, eficiencia subjetiva habitual del sueño, trastornos del sueño, uso de medicación para dormir y disfunción diurna en el último mes. La suma total del ICSP fluctúa entre 0 a 21 , resultando que una puntuación $\leq 11$ se considera 
buen durmiente $y>11$ mal durmiente. La confiabilidad del instrumento ICSP fue 0.865 según la prueba de Alfa de Cronbach. Para la tabulación de los datos recolectados, se realizó la revisión de cada cuestionario y se elaboró una hoja de datos en Microsoft Excel, la cual se ingresó en el programa SPSS versión 22, con el propósito de analizar las variables de estudio. Para describir las variables calidad de sueño, obesidad y circunferencia de cintura se hicieron tablas de frecuencia y porcentajes. Asimismo, para determinar la relación entre las variables de estudio se utilizó la prueba estadística chi cuadrado de Pearson con significancia estadística $\mathrm{p}<0,05$.

\section{Resultados}

En la tabla 1 se muestra la distribución sociodemográfica, con respecto al estado civil. El mayor porcentaje corresponde a los casados, tanto para mujeres $64.7 \%$ y varones $37.8 \%$. En relación al nivel de instrucción, ambos sexos tienen nivel primario: el 71.6\% mujeres y el $51.2 \%$ varones. Por otro lado, el $68.1 \%$ de las mujeres tienen de 1 a 2 hijos; de la misma manera, los varones con un $65.9 \%$. Finalmente, el mayor porcentaje procede de la costa: $56 \%$ mujeres y $53.7 \%$ varones.

Tabla 1

Distribución de la muestra según las variables sociodemográficas.

\begin{tabular}{lcccc}
\hline \multirow{2}{*}{ Ítem } & \multicolumn{2}{c}{ Género } & \multicolumn{2}{c}{ Masculino } \\
& $\mathrm{N}^{\circ}$ & $\%$ & $\mathrm{~N} .^{\circ}$ & $\%$ \\
\hline Estado civil & & & & \\
Soltero & 9 & 7.8 & 11 & 13.4 \\
Casado & 75 & 64.7 & 31 & 37.8 \\
Conviviente & 24 & 20.7 & 30 & 36.6 \\
Viudo & 5 & 4.3 & 4 & 4.9 \\
Separado & 3 & 2.6 & 6 & 7.3 \\
Grado de instrucción & & & & \\
Ninguno & 7 & 6.0 & 8 & 9.8 \\
Primaria & 83 & 71.6 & 42 & 51.2 \\
Secundaria & 24 & 20.7 & 28 & 34.1 \\
Superior & 2 & 1.7 & 4 & 4.9 \\
Número de hijos & & & & \\
Ninguno & 5 & 4.3 & 11 & 13.4 \\
1 a 2 & 79 & 68.1 & 54 & 65.9 \\
3 a 4 & 26 & 22.4 & 14 & 17.1 \\
5 a 6 & 6 & 5.2 & 3 & 3.7 \\
Procedencia & & & & \\
Costa & 65 & 56.0 & 44 & 53.7 \\
Sierra & 27 & 23.3 & 21 & 25.6 \\
Selva & 24 & 20.7 & 17 & 20.7 \\
\hline
\end{tabular}

En la tabla 2 se observa el grado de obesidad según el IMC, el cual refiere que el $41.4 \%$ del género femenino presenta obesidad grado II, y el $32.8 \%$ obesidad grado I. Con respecto al género masculino se muestra un comportamiento similar al sexo femenino, donde el $42.7 \%$ presenta obesidad grado II y el $32.9 \%$ obesidad grado I, y solo el menor porcentaje presenta obesidad grado III en ambos sexos. 
Plaza, K. y Ambulay, J.

Tabla 2

Distribución de la muestra según el grado de obesidad y género.

\begin{tabular}{llcc}
\hline Género & Grado de obesidad & N. ${ }^{\circ}$ & $\%$ \\
\hline \multirow{3}{*}{ Femenino } & Obesidad I & 38 & 32.8 \\
& Obesidad II & 48 & 41.4 \\
& Obesidad III & 30 & 25.9 \\
\multirow{3}{*}{ Masculino } & Total & 116 & 100.0 \\
& Obesidad I & 27 & 32.9 \\
& Obesidad II & 35 & 42.7 \\
& Obesidad III & 20 & 24.4 \\
& Total & 82 & 100.0 \\
\hline
\end{tabular}

En la tabla 3 se clasificó el riesgo cardiovascular alto o aumentado según la circunferencia de la cintura. En ambos sexos, se encontró un mayor porcentaje de riesgo aumentado, un 72.4\% para las mujeres y $67.1 \%$ para los varones. En el caso del riesgo alto, que es mucho más grave, se encontró un menor porcentaje para ambos sexos.

Tabla 3

Distribución de la muestra según los niveles de la circunferencia de la cintura y género.

\begin{tabular}{llcc}
\hline Género & Circunferencia de la cintura & N. ${ }^{\circ}$ & $\%$ \\
\hline \multirow{3}{*}{ Femenino } & Riesgo incrementado & 84 & 72.4 \\
& Riesgo alto & 32 & 27.6 \\
\multirow{3}{*}{ Masculino } & Total & 116 & 100.0 \\
& Riesgo incrementado & 55 & 67.1 \\
& Riesgo alto & 27 & 32.9 \\
& Total & 82 & 100.0 \\
\hline
\end{tabular}

En la tabla 4 se clasificó los niveles de calidad de sueño de los comerciantes por género, en el cual se encontró que ambos sexos tienen mayor porcentaje de mala calidad de sueño, $91.4 \%$ para las mujeres y el $92.7 \%$ para los varones.

Tabla 4

Distribución de la muestra según los niveles de calidad de sueño por género.

\begin{tabular}{llcc}
\hline Género & Niveles de Calidad de Sueño & $\mathrm{n}$ & $\%$ \\
\hline Femenino & Bueno & 10 & 8.6 \\
& Malo & 106 & 91.4 \\
& Total & 116 & 100.0 \\
\multirow{4}{*}{ Masculino } & Bueno & 6 & 7.3 \\
& Malo & 76 & 92.7 \\
& Total & 82 & 100.0 \\
\hline
\end{tabular}

En la tabla 5 se observa que existe una relación estadísticamente significativa entre los grados de obesidad y el nivel de calidad de sueño en comerciantes del género femenino, siendo mayor el número de mujeres con una mala calidad de sueño $(n=106)$ versus la buena calidad de sueño $(n=10)$. Por otro lado, en el género masculino no existe relación estadísticamente significativa entre el grado de obesidad y el nivel de calidad de sueño, sin embargo, también se muestra un elevado número de varones con mala calidad de sueño ( $\mathrm{n}=76)$ frente a una buena calidad de sueño $(n=6)$. 
Relación entre la calidad de sueño con el grado de obesidad y circunferencia de cintura en comerciantes del Gran Mercado Mayorista de Lima, 2016

Tabla 5

Relación entre la calidad de sueño y el grado de obesidad por género.

\begin{tabular}{|c|c|c|c|c|c|}
\hline \multirow[t]{2}{*}{ Género } & \multirow{2}{*}{$\begin{array}{l}\text { Grado de } \\
\text { Obesidad }\end{array}$} & \multicolumn{2}{|c|}{ Calidad de sueño } & \multirow[t]{2}{*}{ Total } & \multirow{2}{*}{$\begin{array}{l}\text { Chi-cuadrado de } \\
\text { Pearson }\end{array}$} \\
\hline & & Buena & Mala & & \\
\hline \multirow[t]{3}{*}{ Femenino } & Obesidad I & $7(70.0 \%)$ & $31(29.2 \%)$ & $8(32.8 \%)$ & $\begin{array}{l}X^{2}=7.80 \\
p=.020\end{array}$ \\
\hline & Obesidad II & $3(30.0 \%)$ & $45(42.5 \%)$ & $48(41.4 \%)$ & \\
\hline & Obesidad III & $0(0.0 \%)$ & $30(28.3 \%)$ & $30(25.9 \%)$ & \\
\hline \multirow{5}{*}{ Masculino } & Total & $10(100.0 \%)$ & $106(100 \%)$ & $116(100.0 \%)$ & $\begin{array}{c}\mathrm{X}^{2}=4.60 \\
\mathrm{p}=.100\end{array}$ \\
\hline & Obesidad I & $1(16.7 \%)$ & $26(34.2 \%)$ & $27(32.9 \%)$ & \\
\hline & Obesidad II & $5(83.3 \%)$ & $30(39.5 \%)$ & $35(42.7 \%)$ & \\
\hline & Obesidad III & $0(0.0 \%)$ & $20(26.3 \%)$ & $20(24.4 \%)$ & \\
\hline & Total & $6(100.0 \%)$ & $76(100.0 \%)$ & $82(100.0 \%)$ & \\
\hline
\end{tabular}

En la tabla 6 se observa que existe una relación significativa $(p=0.041)$ entre la circunferencia de cintura y el nivel de calidad de sueño en los comerciantes del género femenino, siendo mayor el número de mujeres con riesgo aumentado respecto al cardiovascular $(n=74)$ y un menor número al riesgo alto $(n=32)$.

En el género masculino no existe relación estadísticamente significativa entre la circunferencia de cintura y el nivel de calidad de sueño, pero se muestra un número elevado de varones que tienen mala calidad de sueño con riesgo aumentado $(n=50)$ y menor número con riesgo alto $(n=26)$.

Tabla 6

Relación entre la calidad de sueño y circunferencia de la cintura por género.

\begin{tabular}{|c|c|c|c|c|c|}
\hline \multirow[t]{2}{*}{ Género } & \multirow{2}{*}{$\begin{array}{l}\text { Circunferencia de la } \\
\text { cintura }\end{array}$} & \multicolumn{2}{|c|}{ Calidad del Sueño } & \multirow[t]{2}{*}{ Total } & \multirow{2}{*}{$\begin{array}{c}\text { Chi- cuadrado } \\
\text { de Pearson }\end{array}$} \\
\hline & & Buena & Mala & & \\
\hline & Riesgo aumentado & & & & $\begin{array}{c}\mathrm{X}^{2}=4.16 \\
\mathrm{p}=.041\end{array}$ \\
\hline \multirow[t]{4}{*}{ Femenino } & & $10(100.0 \%)$ & $74(69.8 \%)$ & $84(72.4 \%)$ & \\
\hline & Riesgo alto & $0(0.0 \%)$ & $32(30.2 \%)$ & $32(27.6 \%)$ & \\
\hline & Total & $10(100.0 \%)$ & $106(100.0 \%)$ & $116(100.0 \%)$ & \\
\hline & & & & & $\begin{array}{l}X^{2}=.77 \\
p=.379\end{array}$ \\
\hline \multirow[t]{4}{*}{ Masculino } & Riesgo aumentado & & & & \\
\hline & & $5(83.3 \%)$ & $50(65.8 \%)$ & $55(67.1 \%)$ & \\
\hline & Riesgo alto & $(16.7 \%)$ & $26(34.2 \%)$ & $27(32.9 \%)$ & \\
\hline & Total & $6(100.0 \%)$ & $76(100.0 \%)$ & $82(100.0 \%)$ & \\
\hline
\end{tabular}




\section{Discusión}

Con respecto al grado de obesidad, tanto en hombres como en mujeres, se encontró un mayor porcentaje de obesidad grado II seguidamente del grado I. Probablemente esta predominancia del grado II podría ser explicada porque el mayor porcentaje de adultos son de la costa o tienen un nivel de instrucción primaria (Tabla 1), lo cual atribuye a una mayor disponibilidad de alimentos y a un menor conocimiento sobre los buenos hábitos de calidad de vida. En ambos sexos predominó la obesidad tipo II y una mala calidad de sueño. En el estudio de Rahe (Rahe et al. 2015) se reportó una mala calidad de sueño en mujeres de bajo grado de instrucción donde el menor porcentaje era de estado civil casado. Asimismo, se encontró que las mujeres obesas tienen un nivel de instrucción primaria $(71 \%)$, pero en su mayoría son casadas $(74.7 \%)$.

Al evaluar la circunferencia de cintura, que determina el riesgo cardiovascular, se encontró que en ambos sexos existe mayor porcentaje con riesgo incrementado que con riesgo alto (Tabla 2). Además, el riesgo incrementado fue prevalente en la obesidad tipo I y II, y el riesgo alto en la obesidad III. Sin embargo, la asociación de la circunferencia abdominal con la calidad de sueño solo se encontró en las mujeres $(\mathrm{p}=0.041)$. Esto es apoyado por la revisión de Mezick (Mezick, Wing and McCaffery 2013), donde se encontró una mayor frecuencia en la mala calidad de sueño por las pocas horas de sueño, aumentando el riesgo cardiovascular. Además, la mayor circunferencia se asocia con una menor eficiencia del sueño según la prueba de actigrafía. La menor cantidad de horas de sueño (5 horas) favorece a que se ingiera una mayor cantidad de energía y con ello la ganancia de peso. Por otro lado, la disminución de sueño aumenta los niveles de cortisol por el eje-hipotálamo-pituitaria- adrenal, lo cual favorece la distribución de grasa a nivel abdominal (Hewagalamulage et al. 2016; Joseph et al. 2016).

En los comerciantes obesos de ambos géneros, se encontró un elevado porcentaje de mala calidad de sueño, varones $92.7 \%$ y mujeres $91.4 \%$ (Tabla 3 ). Algunos de los ítems que influyó en esta mala calidad de sueño fue el promedio de horas que duermen al día: 5 horas, el no conciliar el sueño durante 3 o más veces por semana (44\% varones y $43 \%$ mujeres) y finalmente el no poder respirar bien (3 o más veces por semana). Este último se relaciona con la apnea obstructiva del sueño, la dificultad para respirar por un colapso de la vía aérea superior, el cual se asocia fuertemente al desarrollo de enfermedades cardiovas- culares (Salvador et al. 2004). En el estudio de Westerlund (Westerlund et al. 2014) también se mostró que tanto varones como mujeres obesas tienen mala calidad de sueño, donde el percentil 90 de ambos sexos afirma que dormían menos de 5 horas, logrando un IMC mayor a 30. En el estudio, también se encontró una relación estadísticamente significativa entre el grado de obesidad y mala calidad de sueño en las mujeres, mientras que en los varones no se observó.

Similarmente, en el estudio de Bidulescu (Bidulescu et al. 2010) se encontró asociación en las mujeres obesas con mala calidad de sueño, pero no en los varones. Dentro de la población las mujeres obesas que duermen menos de cinco horas fueron el mayor porcentaje $(49.4 \%, \mathrm{p}<0.03)$ (Bidulescu et al. 2010). En el estudio de Westerlund (Westerlund et al. 2014) se encontró relación en las mujeres que dormían $<5$ horas con respecto al elevado IMC (obesidad), pero a diferencia del estudio ellos sí encontraron relación en los varones. Esto probablemente se debe a que ellos no lo clasificaron por grados de obesidad y utilizaron una población mayor al estudio $(n=799)$. Una de las explicaciones sería que la restricción del sueño incrementa los niveles de grelina y el péptido YY, disminuyendo los niveles de leptina e insulina, lo que conduce a un aumento del apetito e ingesta de alimentos que conlleva al incremento del IMC como obesidad (Durán and Reyes 2016).

En el estudio de Chang et al (Chang et al. 2013), se encontró que las mujeres obesas con corta duración del sueño se asocian con la mala calidad de sueño, siendo las mujeres más propensas a tener dificultad para dormir que los varones. Esto probablemente se debe a que en el ciclo menstrual de la fase lútea se interrumpe el sueño, de igual manera en la menopausia por los cambios hormonales que también alteran el sueño (Krishnan and Collop 2006). Adicionalmente, las mujeres desempeñan un doble papel, por el trabajo, quehaceres del hogar o cuidar a sus hijos, lo cual disminuye las horas de descanso. En el estudio de Silva (Silva et al. 2008), se encontró que las mujeres tardan más que los varones en iniciar el sueño; por el contrario, el estudio también mostró que las mujeres y los hombres tardan un tiempo similar en iniciar el sueño, de 16 a 30 minutos. Esta diferencia probablemente se debe a que en el estudio de Silva utilizaron una población mayor en varones $(69.5 \%$, 31-60 años).

Frente a todo esto se menciona que no conservar una buena calidad de sueño perjudica la salud por la re- 
lación con la obesidad y riesgo cardiovascular, especialmente las mujeres. Se sabe que la obesidad es una epidemia que está en aumento y las enfermedades cardiovasculares son la primera causa de muerte en el mundo. Estas enfermedades pueden ser transmitidas a la siguiente generación por factores epigenéticos que llevará a un círculo de enfermedades. Por lo tanto, este resultado lleva a tomar conciencia acerca de la calidad de sueño, siendo necesario difundirlo para el beneficio de los semejantes.

\section{Conclusiones}

Existe una relación significativa entre el grado de obesidad y circunferencia de cintura con la calidad de sueño en mujeres, y no en varones, especialmente en las personas que duermen menos de 5 horas. La mayor prevalencia del grado de obesidad fue el tipo II, mientras que en la circunferencia de la cintura el riesgo incrementado fue tanto para varones como para mujeres.

\section{Referencias}

Bidulescu, A. et al. 2010. "Interaction of Sleep Quality and Psychosocial Stress on Obesity in African Americans: The Cardiovascular Health Epidemiology Study (CHES)". BMC public health: 10.

Cárdenas, V., Roandy, G. and Hernández, C. 2012. "El rol del sueño como riesgo de obesidad". Desarrollo Cientif Enferm 48(3): 14-18.

Carmenate, M. et al. 2007. "Obesidad y circunferencia de la cintura en adolescentes madrileños". Revista Cubana de Salud Pública 33(3): 1-9.

Carrillo P., Ramírez J., Magaña K. 2013. "Neurología del sueño y su importancia. Antología para el estudiante universitario". Article 56(4):15.

Cascales, M. 2015. "Obesidad: Pandemia del Siglo XXI". Monografias de la Real Academia Nacional de Farmacia: $14-46$.

Chang, H. et al. 2013. "The Association between Self-Reported Sleep Quality and Overweight in a Chinese Population". Obesity 21(3): 486-92.

Doris Álvarez Dongo, José Sánchez, Guillermo Gómez Guizado, Carolina Tarqui Mamami. 2012. "Sobrepreso y obesidad: Prevalencia y determinantes sociales del exceso de peso en la población peruana". Rev. Perú Med. Exp. Salud Pública 29(3): 303-13.

Durán, S. and Reyes, H. 2016. "Relación entre cantidad de sueño nocturno y obesidad en adultos mayores chilenos". 66(4): 142-48.
Granados, Z. et al. 2013. "Calidad del sueño en una facultad de medicina de Lambayeque". Article 4(74): 311-14.

Guevara, Y. 2013. "Factores psicosociales y su relación con la calidad de sueño en estudiantes de enfermería de la UNJBG, Tacna, 2012.

Hewagalamulage, S., Lee, T., Clarke, I. and Henry, B. 2016. "Stress, cortisol and obesity: a role for cortisol responsiveness in identifying individuals prone to obesity". Domestic animal endocrinology 56: 112-20.

Instituto Nacional de Estadística e Informática. 2016. “Población peruana padece de obesidad".

Joseph, J. et al. 2016. "Features: The multi ethnic study of atherosclerosis". Metabolism 68: 95-107.

Krishnan, V., and Collop, N. 2006. "Gender differences in sleep disorders". Current opinion in pulmonary medicine 12(6): 383-89.

Mezick, E., Wing, R. and McCaffery, J. 2013. "Associations of self reported and actigraphy assessed sleep characteristics with body mass index and waist circumference in adults: moderation by gender". Sleep Medicine 15(1): 64-70.

Moreno, M. 2012. "Definición y clasificación de la obesidad". Revista médica Clínica Las Condes 23(2): $124-28$.

Nutrición. Instituto Nacional de Salud - Centro Nacional de Alimentación. 2016. "Desnutrición y obesidad en nuestro país".

OMS. 2015. "Obesidad y sobrepeso". Organización Mundial de la Salud. http://www.who.int/mediacentre factsheets/fs311/es/.

Rahe, C., Czira, M., Teismann, H. and Berger, K. 2015. "Associations between poor sleep quality and different measures of obesity”. Sleep Medicine 16(10):15.

Salvador, Javier et al. 2004. "The obstructive sleep apnoea syndrome in obesity: a conspirator in the shadow". Revista de medicina de la Universidad de Navarra 48(2):55-62.

Silva, A. et al. 2008. "Gender and age differences in polysomnography findings and sleep complaints of patients referred to a sleep laboratory". Brazilian Journal of Medical and Biological Research 41(7): 1067-75.

Westerlund, A. et al. 2014. "Habitual sleep patterns and the distribution of body mass index: cross sectional findings among swedish men and women". Sleep Medicine 15(10): 1196-1203. 